\title{
EFFECTS OF WIND CLUMPING ON COLLIDING WINDS
}

\author{
SÉBASTIEN LÉPINE \\ Département de Physique, Université de Montréal, C.P. 6128, Succ. A, Montréal, QC, \\ H3C 3J7, Canada, and Observatoire du Mont-Mégantic
}

\begin{abstract}
Recent studies of variable Wolf-Rayet emission lines reveal a hierarchy of structures, characterized by power laws analogous to what is expected from supersonic compressible turbulence. The collision of inhomogeneous winds can be very different from the case of smooth winds. The difference will mainly depend on two factors: (i) the relative importance of the inhomogeneous compared to the homogeneous component; and (ii) the characteristic filling factor of the inhomogen eous component. Using relations derived from observations of variable line structures ("blobs"), it can be deduced that the flux emitted by the inhomogeneous part of the wind of a WR star is dominated by its smallest structures. This implies that a significant fraction of the underlying emission line profile could be produced by small, undetectable inhomogeneities. It can also be deduced that the volume spanned by the inhomogeneities is dominated by the largest structures. This in turn implies that the filling factor should be low, or that we are dealing with a fractal-like hierarchy. It is suggested that the wind is composed of dense structures separated by large "voids" which may actually be filled by a homogeneous wind component. The interacting zone of two inhomogeneous colliding winds should thus be much more extended in space than for a smooth-wind model, because the dense, inhomogeneous structures are able to penetrate through the large "voids".
\end{abstract}

Key words: stars: Wolf-Rayet - winds - clumping

\section{Inhomogeneous and colliding winds}

Models of colliding winds are made on the assumption that they are smooth and homogeneous ( $c f$. Stevens et al. 1992). However, much evidence exists to support the idea of inhomogeneous winds, and some effects of inhomogeneity on colliding winds have already been studied by Cherepashchuk (1990). Recent work by Robert (1992), Moffat et al. (1993), Moffat \& Robert (1994) and Lépine (1994), suggests that WR winds (and possibly all hot-star winds) are dominated by an inhomogeneous component, which can be described by scaling laws analogous to those for compressible supersonic turbulence (Henriksen 1991). This could result in important consequences for the collision of two inhomogeneous winds.

The behavior of colliding inhomogeneous winds will strongly depend on the filling factor. If this factor is high (i.e., inhomogeneities occupy most of the space), this will allow frequent collisions between dense structures, and will produce a bow-shock like that produced by smooth winds. This shock zone may be more unstable, however, because it originates from flows with densities that already vary on short timescales. On the other extreme, a low filling factor will lead to large empty spaces between the "blobs", thus reducing "colliding effects". An example of an extreme case is given by two colliding galaxies, where the stellar (inhomogeneous) component is barely 
affected, while the gaseous (relatively homogeneous) component is strongly interacting. If a homogeneous component exists, it will form a bow-shock as modeled by Stevens et al. (1992). In that case, dense inhomogeneities should be able to run through the shock zone, as suggested by Cherepashchuk (1990).

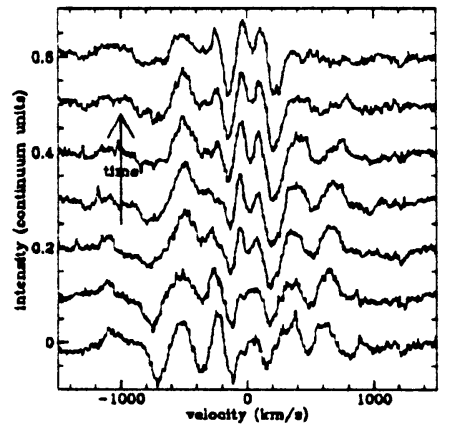

Fig. 1. Difference from the mean spectra of one night's data for the flat top CIII $5696 \AA$ emission line $(\mathrm{FWHM} \sim 3000 \mathrm{~km} / \mathrm{s})$ of WR137 (WC7+O). Variable features can be traced from one spectrum to the next. Individual structures are extracted and their physical parameters analysed, giving the relations described in the text.

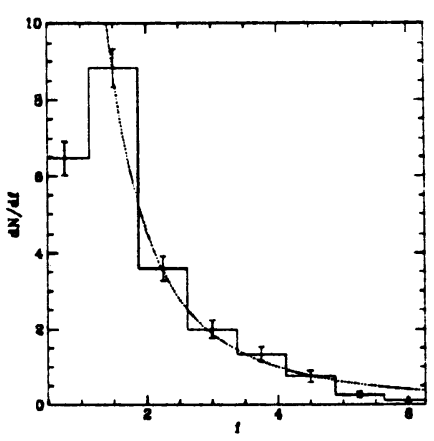

Fig. 2. Histogram showing the average number of structures detected as a funtion of flux (arbitrary units). The lower the flux, the more numerous they are. The dotted line shows a power law $d N \sim f^{-2.2} d f$. The presence of instrumental noise at the smallest scales leads to a finite detection threshold.

\section{Modelling the inhomogeneous component}

A semi-empirical model is elaborated from observations of variable features in emission lines (see Figure 1 for an example) of five WR stars, taken from the work of Robert (1992), three of them being in binary systems: WR135 (WC8), WR136 (WN6), WR137(WC7+abs(O?)), WR138 (WN5+abs(O?)), WR140 (WC7+04). Intrinsic wind features are extracted (cf. Lépine 1994), and their physical parameters determined. One finds the following relations: a scaling law between flux and velocity dispersion $f \sim \sigma_{v}^{\beta}$ (see Fig. 2 of the poster by Lépine, these proceedings), and a flux spectrum $d N \sim f^{\alpha} d f$ (Figure 2). These relations are very similar for all five stars. Moreover, using the flux relation for recombination emission lines $f \sim \rho^{2} l^{3}$, where $\rho$ is the density and $l$ the spatial dimension (the temperature of line formation is assumed $\sim$ constant), and the relation $\rho \sim 1 / l$, as for a supersonic compressible turbulent medium ( $c f$. Moffat \& Robert, 1994), one finds: $d N \sim f^{\alpha} d f(1), f \sim \sigma_{v}^{\beta}$ (2), and $l \sim \sigma_{v}^{\gamma}$ (3), where $\alpha$ and $\beta$ are determined directly from the observations, and $\gamma$ is obtained from theoretical considerations. We define $F_{i n h}$, 
the total flux emitted by the inhomogeneous component and $V_{i n h}$, the total volume occupied by the inhomogeneous structures. Then, $d F_{i n h} \sim f d N$ and $d V_{i n h} \sim l^{3} d N$. By combining this with (1), (2) and (3), one finds the following relations: $d F_{i n h} \sim f^{\alpha+1} d f(4)$ and $d V_{i n h} \sim f^{(3 \gamma / \beta+\alpha)} d f$ (5). From observations, $\alpha \simeq-2.5$ and $\beta \simeq 2.0$. The compressible turbulence hypothesis gives $\gamma=2$.

\section{Importance of the inhomogeneous component}

We now make the assumption that relations (4) and (5) are valid over small (undetectable) scales as well as over large scales.

Relation (4) gives $d F_{i n h} \sim f^{-1.5} d f$ and is solely dependant on the observations. It implies that the total flux is dominated by the contribution of the smallest structures. This means that the observed variable features may only be the tip of the iceberg, and that a significant part of the global underlying profile could be due to a large number of small undetectable inhomogeneities.

Relation (5) gives $d V_{i n h} \sim f^{0.5} d f$, and implies that the volume occupied by the "blobs" is dominated by the largest structures. This means that small structures are not numerous enough to fill the space between large structures, or they are part of large structures (i.e. fractal-like geometry). In either case, it is safe to assume that there will be large amounts of "empty" space between large structures; the filling factor will be small. These "voids" could be filled by a homogeneous component, whose importance relative the the inhomogeneous part cannot be estimated as yet.

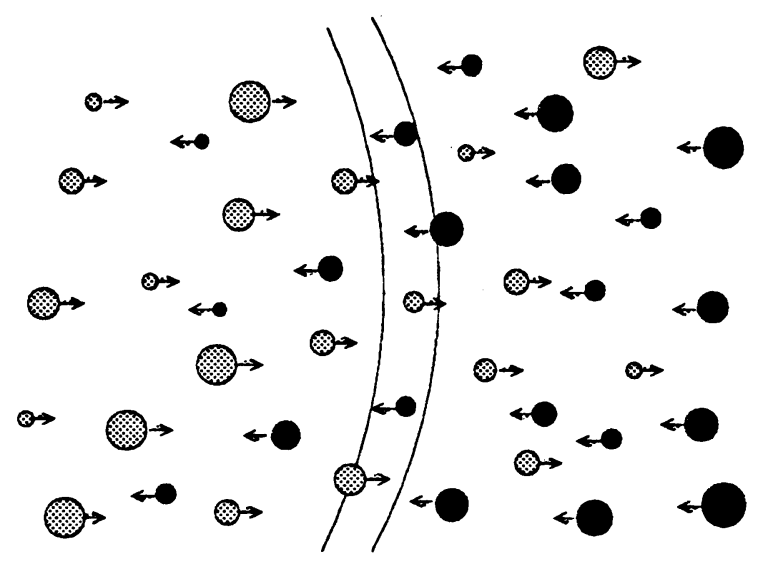

Fig. 3. Suggested model for clumped colliding winds. The inhomogeneous component ("blobs") does not take part in the formation of the shock-zone (center) by the homogeneous component. 


\section{Conclusion}

From scaling relations observed for inhomogeneous structures detected in the wind of five WR stars, and using supersonic compressible turbulence as a hypothesis of their their origin, the inhomogeneous component of the wind can be characterized. This component may be dominant and influence greatly the behaviour of colliding winds. The filling factor is found to be low; a dense structure from the wind of one star in a binary could penetrate deep through the wind of the other star by travelling through the large "voids" between the "blobs" (cf. Figure 3). If an homogeneous component exists and is creating a bow-shock, the dense blobs could pass clear through the shock zone (cf. Cherepashchuk 1990).

Generally speaking, wind clumping will result in an interacting region occupying a larger volume. Also, the shock zone produced by the homogeneous component should be thinner, since part of the wind's mass is already taken up by the inhomogeneous (and less interacting) part. The importance of this effect will thus be strongly dependent on the fraction of mass contained in the inhomogeneous part of the wind, which remains to be evaluated.

\section{References}

Cherepashchuk, A.M. 1990, Astron. Zh. 67, 955 (=Sov. Astron. 34, 481)

Henriksen, R.N. 1991, ApJ 377, 500

Lépine, S. 1994, in: A.F.J. Moffat, S.P. Owocki, A.W. Fullerton \& N. St-Louis (eds.), Instability and Variability of Hot-Star Winds, Ap Space Sci. in press

Moffat, A.F.J., Lépine, S., Henriksen, R.N., Robert, C. 1994, in: A.F.J. Moffat, S.P. Owocki, A.W. Fullerton \& N. St-Louis (eds.), Instability and Variability of Hot-Star Winds, Ap Space Sci. in press

Moffat, A.F.J., Robert, C. 1994, ApJ 421, 310

Robert, C. 1992, PhD thesis, Université de Montréal

Stevens, I.R., Blondin, J.M., Pollock, A.M.T. 1992, ApJ 386, 265 


\section{DISCUSSION:}

Pollock: You didn't actually tell us what the filling factor of blobs actually is? Do you have any idea how it compares with the flux in blobs and flux in the smooth line?

Lepine: The exact filling factor is dependent on the size of the largest blobs. Assuming a radius of about $1 \mathrm{R}_{\mathrm{o}}$ for these blobs, I calculated that the filling factor could be at most 0.01 . The flux is dominated by the smaller blobs, and they are so numerous that the resulting profile is smooth. In that case, it becomes impossible to distinguish them from the homogenous component, and any comparison is tricky.

Cherepashchuk: It is very important to observe X-ray from colliding winds from $\mathrm{WR}+\mathrm{O}$ binaries at short timescales (from $\sim 100 \mathrm{sec}$ to several hours). Such observations could give us direct information about the clumping structure of WR wind (density jumps, filling factor, distribution of the characteristic masses and dimensions of blobs etc).

Shaviv: Coming back to your picture of an American football team, I can hardly imagine that even an American football player will survive a collision at $\sim 1000 \mathrm{~km} / \mathrm{sec}$ relative velocity (or even higher velocity) let alone poorly held and loose clumps of plasma. Thus I can hardly see, or understand, the picture of a shock under these conditions and the survival of clumps thereof. Lepine: What I meant by this illustration is that if the filling factor is high, then collisions between the clump would be frequent. Eventually, if the flows are, on average, constant in time, an equilibrium will be attained and an interface will be formed separating the winds. This interface will be well defined because a blob is not able to go very far through the opposite wind. Owocki: Analogies with "compressible turbulence" from giant molecular clouds are fine, but there exist direct calculations of line-driven instabilities that are likely to produce this clumping. These show that the high density regions are highly compressed by supersonic shocks of highspeed, rarified material. The density contract between the high and low density can be very large indeed, at least a factor ten, and often much more (X1000!). It has not yet been possible to do these simulations in 3-D but I think nonetheless the 1-D simulations done so far provide strong indicators about such questions regarding filling factor and density contrast as raised here.

Brown: Do you think of the homogeneous wind component as having high or low density, and what is its relation to the turbulent blob component in terms of pressure balance?

Lepine: I used the assumptions that $\rho \sim 1 / l$, and this is predicted by compressible turbulence theory. In that case, the density of the smallest blobs could be very high, and this can become a problem for pressure balance. However, it showed that it is not realistic to extrapolate this relation to very small scales, because the flux then becomes infinite. That is why I suggest that the physics of the smallest scales can be different and the assumptions of pressure equilibrium could be reconsidered. 